\title{
Changes in Flexibility and Force are not Different after Static Versus Dynamic Stretching
}

\section{(ㄷ) (i) 8}

\author{
Authors \\ Shingo Matsuo', Masahiro Iwata1, 2, Manabu Miyazaki³ ${ }^{3}$ Taizan Fukaya ${ }^{4}$, Eiji Yamanaka ${ }^{5}$, Kentaro Nagata ${ }^{6}$, \\ Wakako Tsuchida1, 2, Yuji Asai' ${ }^{1}$, Shigeyuki Suzuki ${ }^{7}$
}

\section{Affiliations}

1 Department of Rehabilitation, Faculty of Health Sciences, Nihon Fukushi University, Handa, Japan

2 Department of Physical and Occupational Therapy, Nagoya University Graduate School of Medicine, Nagoya, Japan

3 Department of Physical Therapy, Faculty of Medical Science for Health, Teikyo Heisei University, Toshima-ku, Japan

4 Department of Rehabilitation, Kyoto Kujo Hospital, Kyoto, Japan

5 Department of Rehabilitation Medicine, Tokyo Bay Rehabilitation Hospital, Narashino, Japan

6 Department of Rehabilitation, Kariya Toyota General Hospital, Kariya, Japan

7 Department of Health and Sports Sciences, School of Health Sciences, Asahi University, Mizuho, Japan

Key words

warm-up exercise, range of motion, passive torque, passive stiffness, muscle force

received $\quad 03.04 .2019$

revised $\quad 06.08 .2019$

accepted $\quad 07.08 .2019$

\section{Bibliography}

DOI https://doi.org/10.1055/a-1001-1993

Sports Medicine International Open 2019; 3: E89-E95

(c) Georg Thieme Verlag KG Stuttgart · New York

ISSN 2367-1890

\author{
Correspondence \\ Dr. Shigeyuki Suzuki \\ Department of Health and Sports Sciences \\ School of Health Sciences \\ Asahi University \\ 1851 Hozumi, Mizuho \\ 501-0296 Gifu \\ Japan \\ Tel.: + 81/58/329 1178 \\ suzuki@met.nagoya-u.ac.jp
}

\begin{abstract}
In this study, we examined the effects of static and dynamic stretching on range of motion (ROM), passive torque (PT) at pain onset, passive stiffness, and isometric muscle force. We conducted a randomized crossover trial in which 16 healthy young men performed a total of $300 \mathrm{~s}$ of active static or dynamic stretching of the right knee flexors on two separate days in random order. To assess the effects of stretching, we measured the ROM, PT at pain onset, passive stiffness during passive knee extension, and maximum voluntary isometric knee flexion force using an isokinetic dynamometer immediately before and after stretching. Both static and dynamic stretching significantly increased the ROM and PT at pain onset $(p<0.01)$ and significantly decreased the passive stiffness and isometric knee flexion force immediately after stretching $(p<0.01)$. However, the magnitude of change did not differ between the two stretching methods for any measurements. Our results suggest that $300 \mathrm{~s}$ of either static or dynamic stretching can increase flexibility and decrease isometric muscle force; however, the effects of stretching do not appear to differ between the two stretching methods.
\end{abstract}

\section{Introduction}

Static stretching (SS) is commonly performed to improve flexibility and as a component of warm-up exercises in the belief that it will reduce the risk of injury [26, 31]. Many studies have shown that SS improves flexibility as measured by tests of range of motion (ROM), passive torque (PT), and passive stiffness $[7,16,18]$. However, re- cent review articles reported that prolonged ( $>30-60 \mathrm{~s}$ ) SS can have detrimental effects on muscle performance $[3,13,25]$. Therefore, it may not be advisable to engage in prolonged SS prior to high-level or competitive athletic or training activities [3]. In contrast to SS, dynamic stretching (DS) has recently been recommended as a component of warm-up exercises conducted prior to en- 
gaging in athletic activities. Many recent studies have shown that DS improves muscle power, jump height, and sprint time, and DS has been found to have a more beneficial effect on performance than SS [14, 22, 32].

In terms of muscle performance, the available data appear to indicate that DS is more suitable for warming up compared with SS. However, which stretching method is more effective with respect to improving or maintaining flexibility remains unclear [2]. For example, Behm et al. [4] reported no difference between the effects of SS versus DS on the outcome of the sit and reach test. However, Paradisis et al. [21] reported that the effect of SS on the outcome of the sit and reach test was greater than that of DS in adolescent boys and girls. In contrast, Amiri-Khorasani and Kellis [1] reported that the effect of DS on flexibility was greater than that of SS. Previous studies have shown that lower levels of muscle flexibility [30] and higher levels of stiffness [28] are associated with a higher risk of muscle injury. Thus, stretching prior to engaging in athletic activity has multiple benefits, including reducing the risk of injury and improving athletic performance. However, although both static and dynamic stretches are performed as components of warm-up exercises, few studies have directly compared the acute effects of static and dynamic stretching in terms of flexibility parameters (e. g. range of motion, passive torque at onset pain, and passive stiffness) and muscle force. A comparison of the effects of these two types of stretching on flexibility and muscle force could indicate which stretching methods are most suitable for warming up before engaging in athletic activity.

In this study, we sought to compare the effects of SS and DS on ROM, PT at the onset of pain, passive stiffness, and isometric muscle force. We hypothesized that DS would be more effective than SS in terms of enhancing muscle performance. Moreover, we hypothesized that the effects of DS on flexibility parameters would be equal to or greater than the effects of SS under the same stretching conditions.

\section{Materials and Methods}

\section{Study design}

We conducted a randomized crossover trial. The participants completed measurement sessions on two separate days, one for each stretching type. Specifically, they completed either SS or DS of the right hamstrings for a 300 s period. The order of stretching type was randomized. We obtained the ROM of passive knee extension, PT at the onset of pain, passive stiffness, and maximum voluntary isometric knee flexion force immediately before and after stretching. All participants attended a familiarization session before the first testing day. All measurements were taken at the same time of day $( \pm 1 \mathrm{~h})$.

\section{Participants}

Sixteen healthy young men voluntarily participated in this study (mean \pm standard deviation (SD): aged $22.2 \pm 1.2 \mathrm{y}$, height $170.7 \pm 6.2 \mathrm{~cm}$, body mass $64.0 \pm 11.5 \mathrm{~kg}$, body mass index $21.9 \pm 3.3 \mathrm{~kg} / \mathrm{m}^{2}$ ). All participants were informed regarding the study purpose and protocol and provided written informed con- sent. The study was approved by the Human Research Ethics Committee of our institution (approval number: 14-23). Moreover, this study was performed in accordance with the ethical standards of the International Journal of Sports Medicine [8]. The inclusion criteria were healthy males aged approximately 22 years. The exclusion criteria were lower extremity joint contractures, history of surgical operation on the back or lower extremities, neurological disorders, current regiment of hormones or muscle-affecting drugs, ability to completely extend the right knee from a sitting position as described below (i. e., exceptional flexibility), engagement in competitive sports, regular resistance, aerobics, or flexibility training. The participants were asked to refrain from vigorous physical activity during the experimental period.

\section{Procedures}

\section{Static stretching}

For SS, each participant assumed a standing upright position and placed his right heel (with an extended leg) on a platform $50 \mathrm{~cm}$ high. The participant then reached forward with their arms toward the extended leg while maintaining a proper lordotic curve [4] ( $\triangleright$ Fig. 1a). SS was performed at a tolerable intensity without pain $[11,16,17]$. Ten 30 -s sets of SS were performed with a 20 -s rest period between each set.

\section{Dynamic stretching}

For DS, each participant assumed a standing upright position beside parallel bars and held a parallel bar with his left hand for stability. To stretch the hamstrings, the participants intentionally contracted the right hip flexors with the knee extended and flexed their right hip joint so that their right leg swung up to the anterior aspect of their body [10, 32] ( Fig. 1b). The participants performed this dynamic movement every $2 \mathrm{~s}$. Each exercise was performed 5 times slowly to practice, and then 10 times as quickly as possible without bouncing [10,32]. Ten 30-s sets of DS (15 repetitions of the DS movement in each set) were performed with a 20 -s rest period between each set.

\section{Dependent variables}

We first measured the torque-angle relationship (ROM, PT at pain onset, and passive stiffness) and then measured the isometric muscle force immediately before and after stretching. All dependent variables were obtained using an isokinetic dynamometer (PrimusRS; BTE Technologies, Hanover, MD, USA). The torque and angle signals from the dynamometer were subjected to analog-todigital conversion (PL3508 PowerLab 8/35; ADInstruments, Sydney, Australia) and stored in a personal computer.

Range of motion, passive torque at the onset of pain, and passive stiffness

Measurements were taken with the participant in a sitting position with his hip joint flexed ( $\vee$ Fig. 1c) $[11,16,17]$. Each participant was seated on a chair with the seat tilted maximally and a wedgeshaped cushion inserted between the trunk and the backrest. The participant's chest, pelvis, and right thigh were stabilized with Velcro straps. The knee joint was aligned with the axis of rotation of the dynamometer, and the lever arm attachment was placed just 
proximal to the malleolus medialis. In this position, the average angles of hip and knee flexion were $107.6 \pm 2.3^{\circ}$ and $111.2 \pm 1.8^{\circ}$, respectively. With the participant sitting in the chair ( $\nabla$ Fig. 1c), his knee was extended passively at $5 \%$ to the point of maximum knee extension just before the onset of pain. Torque was recorded continuously during passive knee extension $[11,16,17]$. ROM (in ${ }^{\circ}$ ) was defined as the maximum knee extension angle from the initial position $\left(0^{\circ}\right)$, and PT at the onset of pain (in $\mathrm{Nm}$ ) was defined as the torque at the onset of pain $[11,17]$. Passive stiffness (in Nm/ ${ }^{\circ}$ ) was defined as the slope of the regression line calculated from the torque-angle relationship using the least squares method $[11,16,17]$. Stiffness was calculated using the same knee extension angle range before and after stretching, and the calculated knee extension angle range was defined as the angle from the $50 \%$ maximum knee extension angle to the pre-stretching maximum knee extension angle.

\section{Isometric muscle force}

Isometric muscle force (in $\mathrm{Nm}$ ) was measured in the same position as that used to measure the torque-angle relationship $[11,16,17]$ ( $\triangleright$ Fig. 1c). The participants were instructed to sit with their arms crossed in front of their chest, and to generate maximum knee flexion force for $3 \mathrm{~s}$. They did this three times with a 45 -s rest period between trials [17]. Peak torque was obtained from each contraction, and the average of the three trials was used for further analysis.

\section{Test-retest reliability}

We confirmed test-retest reliability values for all dependent variables by calculating intra-class correlation coefficients (ICCS) and coefficients of variation (CVs). Prior to the data collection in the present study, we conducted a pilot study to examine the test-retest reliability for all dependent variables. The participants were 12 men. The two tests were performed on two separate days and at the same time of the day $( \pm 1 \mathrm{~h})$. We calculated ICC and CV, and the
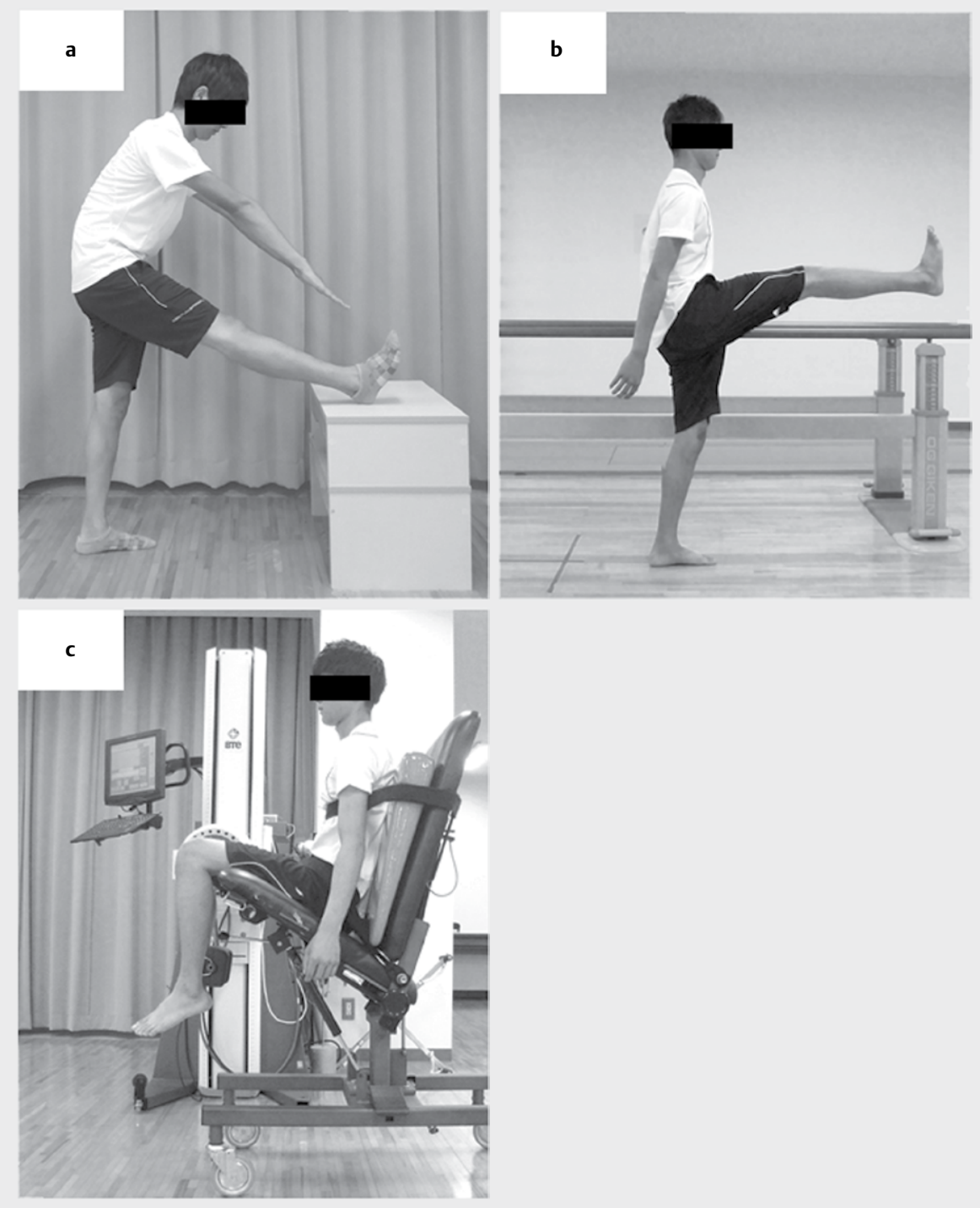

- Fig. 1 Positioning for a static stretching and $\mathbf{b}$ dynamic stretching of the right hamstring. $\mathbf{c}$ Positioning for the measurements of all dependent variables. 
results of these assessments showed that reliability was acceptable for all measures (ROM: 0.903 (ICC), 2.5\% (CV); PT at the onset of pain: $0.934,3.4 \%$; passive stiffness: $0.910,5.7 \%$; isometric muscle force: $0.915,4.2 \%)$.

\section{Statistical analyses}

We determined the number of participants by conducting a sample size estimation using data from the literature [17] and $G$ * Power software (v 3.0.10; Franz Faul, Kiel University, Kiel, Germany). The effect size of $300 \mathrm{~s}$ of static stretching on knee extension ROM, which was calculated from previous data [17], was 0.91599. On the basis of the effect size, $\alpha$ level of 0.05 , and a power $(1-\beta)$ of 0.80 , the minimum number of participants was estimated to be 12 . To strengthen the power of the study, we recruited 16 participants.

We assessed the normality of the data using the Shapiro-Wilk test. This test showed that the ROM and isometric muscle force were normally distributed, but the other parameters were not. Thus, we applied non-parametric tests to all absolute values and relative changes (in \%). We performed the Wilcoxon signed-rank test to identify significant differences between the two stretching methods at each time point as well as the relative change, or the difference from the pre-stretching value. Instead of Cohen's d, we used the r effect size (ES) to calculate the ES for the change from pre- to post-stretching and the between-post-stretching comparison (absolute value and relative change respectively). The $r E S$ was calculated by dividing the Wilcoxon $Z$ score by the square root of the sample size $(r=Z / \sqrt{N})[29]$. This ES was interpreted as follows: small effect, $\geq 0.1$; medium effect, $\geq 0.3$; and large effect, $\geq 0.5$ [29]. Analyses were performed using IBM SPSS statistics version 21.0 (IBM Corp., Armonk, NY, USA), and significance was set at $p<0.05$. All results are expressed as mean $\pm S D$.

\section{Results}

\section{Range of motion}

ROM increased significantly after both SS and DS $(p<0.01)(\triangleright$ Table 1). The ES values for the pre- to post-stretching change were large (SS:
0.88, DS: 0.88). However, we observed no significant differences between SS and DS for pre-stretching values, post-stretching values, or relative change. Moreover, the ES values for the betweenpost-stretching comparison reflected a medium ES (absolute value: 0.45 , relative change: 0.35 ).

\section{Passive torque at the onset of pain}

PT at the onset of pain increased significantly after both SS and DS $(p<0.01)$ ( $\triangleright$ Table 1). The ES values for the pre- to post-stretching change reflected a large ES (SS: 0.88 , DS: 0.88 ). However, we observed no significant differences between SS and DS for pre-stretching values, post-stretching values, or relative change. Moreover, the ES values for the between-post-stretching comparison reflected a small or medium ES (absolute value: 0.23 , relative change: 0.40 ).

\section{Passive stiffness}

Passive stiffness decreased significantly after both SS and DS $(p<0.01)$ ( $>$ Table 1). The ES values for the pre- to post-stretching change reflected a large ES (SS: 0.87 , DS: 0.87 ). However, we observed no significant differences between SS and DS for pre-stretching values, post-stretching values, or relative change. Moreover, the ES values for the between-post-stretching comparison reflected a negligible or small ES (absolute value: 0.00 , relative change: 0.22 ).

\section{Isometric muscle force}

Isometric muscle force decreased significantly after both SS and DS $(p<0.01)$ ( $\vee$ Table 1). The ES values for the pre- to post-stretching change reflected a large ES (SS: 0.88 , DS: 0.88 ). However, we observed no significant differences between SS and DS for pre-stretching values, post-stretching values, or relative change. Moreover, the ES values for the between-post-stretching comparison reflected a negligible ES (absolute value: 0.07 , relative change: 0.01 ).

\section{Discussion}

In this study, we compared the effects of SS and DS on ROM, PT at the onset of pain, passive stiffness, and isometric muscle force. We had hypothesized that DS would be more effective than SS in terms

- Table 1 Effects of stretching on changes in dependent variables.

\begin{tabular}{|c|c|c|c|c|c|}
\hline Dependent variable & Stretching method & Pre & Post & $\begin{array}{l}\text { Relative change } \\
(\%)\end{array}$ & $\begin{array}{l}\text { Intra-condition } \\
\text { difference }\end{array}$ \\
\hline \multirow[t]{3}{*}{$\operatorname{ROM}\left({ }^{\circ}\right)$} & Static stretching & $87.1 \pm 6.6$ & $101.5 \pm 6.5$ & $116.7 \pm 3.4$ & $p<0.01$ \\
\hline & Dynamic stretching & $86.9 \pm 8.1$ & $99.8 \pm 6.6$ & $115.2 \pm 5.9$ & $\mathrm{p}<0.01$ \\
\hline & Inter-condition difference & $p=0.96$ & $p=0.07$ & $p=0.16$ & - \\
\hline \multirow[t]{3}{*}{ PT at the onset of pain (Nm) } & Static stretching & $32.3 \pm 7.2$ & $38.9 \pm 9.0$ & $120.4 \pm 6.5$ & $\mathrm{p}<0.01$ \\
\hline & Dynamic stretching & $32.3 \pm 6.2$ & $37.7 \pm 6.0$ & $117.7 \pm 12.3$ & $\mathrm{p}<0.01$ \\
\hline & Inter-condition difference & $p=0.41$ & $p=0.35$ & $p=0.11$ & - \\
\hline \multirow[t]{3}{*}{ Passive stiffness $\left(\mathrm{Nm} /{ }^{\circ}\right)$} & Static stretching & $0.433 \pm 0.123$ & $0.385 \pm 0.112$ & $89.0 \pm 7.9$ & $p<0.01$ \\
\hline & Dynamic stretching & $0.430 \pm 0.097$ & $0.379 \pm 0.088$ & $88.2 \pm 5.9$ & $p<0.01$ \\
\hline & Inter-condition difference & $p=0.92$ & $p=1.00$ & $p=0.38$ & - \\
\hline \multirow[t]{3}{*}{ Isometric muscle force (Nm) } & Static stretching & $69.2 \pm 15.8$ & $59.7 \pm 15.6$ & $85.7 \pm 8.6$ & $\mathrm{p}<0.01$ \\
\hline & Dynamic stretching & $69.9 \pm 13.5$ & $60.3 \pm 14.3$ & $85.8 \pm 8.2$ & $\mathrm{p}<0.01$ \\
\hline & Inter-condition difference & $p=0.23$ & $p=0.80$ & $p=0.96$ & - \\
\hline
\end{tabular}


of enhancing muscle performance and that the effects of DS on flexibility parameters would be equal to or greater than the effects of SS under the same stretching conditions. However, contrary to expectations, we found that SS and DS did not differ in terms of their affects on ROM, PT at the onset of pain, passive stiffness, or isometric muscle force.

We found that 300 s of SS increased ROM and PT at the onset of pain and decreased passive stiffness and isometric muscle force. These changes were similar to those previously reported after long periods ( $\geq 180 \mathrm{~s}$ ) of SS $[11,16,17]$. Therefore, these effects were as expected. However, contrary to our expectations, a total of $300 \mathrm{~s}$ of DS significantly decreased isometric muscle force in a way that was similar to that elicited by SS. Many previous studies have reported that DS improved performance parameters [14, 22, 32]. In contrast, some review articles have stated that SS decreased the maximum muscle force and performance $[3,13,25]$. A decrease in muscle force and performance after SS might be caused by a reduction in a neural drive, such as a central drive [27], as well as a reduction in peripheral electromyographic activity $[6,12]$. Further, this decrease could be due to a reduction in peripheral force-generating capacity, such as that caused by musculotendinous stiffness, and associated changes in the muscle length-tension relationship [6, 23]. Previous studies have considered how muscle stretching might theoretically reduce the force transfer efficiency from the contractile component to the skeleton alongside stretchinduced reductions in muscle stiffness, although this possibility has not been assessed directly in humans [2]. Our data indicate that the effects of static and dynamic stretching on these variables might be similar. Specifically, our findings suggest that the decrement in isometric muscle force after DS was partly caused by the decrement in passive stiffness, as is the case following SS. However, we did not measure changes in neurophysiological factors using electromyography or other techniques. We also did not measure changes in the performance of muscle contractions such as the rate of force development (contraction speed). Further studies are needed to examine the detailed factors that might lead to decreased isometric muscle force after stretching and the effects of stretching on musculoskeletal biomechanics.

For the SS in the present study, participants performed 10 sets that were 30 -s long and were separated by a 20 -s rest period. They were asked to stretch at an intensity that was below the threshold for pain. For the DS in the present study, participants performed 10 sets that were 30 -s long and were separated by a 20 -s rest period. Each 30 -s set contained 15 repetitions of the DS movement, which involved contracting antagonist muscle groups. The participants were asked to conduct the movements at an intensity that was below the pain threshold. Thus, in both the SS and DS conditions, we asked participants to engage in stretching for a total of 300 s per day. We selected this duration because previous studies showed that $300 \mathrm{~s}$ of static stretching at a tolerable intensity and without pain significantly increased ROM and PT, and decreased passive stiffness and isometric muscle force after stretching $[16,17]$. Additionally, the use of these parameters in the present study enabled us to compare the present data with those from these previous studies.

Yamaguchi and Ishii [33] suggested that explosive performance might be impaired as the volume of DS increases. Therefore, the
DS protocol in the present study might have induced a loss of force rather than improving muscle performance because the total number of DS repetitions was excessive (150 repetitions in total). However, another review reported that greater improvements in peak force and power were observed when longer durations of DS, such as $90 \mathrm{~s}$, were performed [3]. These inconsistent results might have been caused by variability among studies, which is supported by another systematic review article that suggested that it was difficult to demonstrate a dose-response relationship with respect to DS [2]. Herda et al. [9] reported that 430 -s sets of DS in which agonist muscle groups were contracted significantly decreased isometric muscle force. These findings suggest that the characteristics of the DS protocol, such as the number of repetitions, intensity, and type of contraction influence the effects of the protocol on muscle force and performance. Therefore, further studies are required to investigate how differences in DS protocols might affect muscle force and performance.

In terms of flexibility, this study revealed that both SS and DS increased ROM and PT at the onset of pain and that they both decreased passive stiffness. Moreover, the changes in these dependent variables were not different between SS and DS. Mizuno et al. [18] reported that an increase in ROM immediately after SS was attributable to an increase in PT at the onset of pain and a decrease in passive stiffness. These findings suggest that, as with SS, the increase in ROM after DS was caused by the changes in PT at the onset of pain and passive stiffness. Nakamura et al. [20] revealed that a total of 300 s of SS decreased muscle-tendon unit stiffness and muscle stiffness, and that the decrease in muscle-tendon unit stiffness was due to the decrease in muscle stiffness. Therefore, the decrease in passive muscle-tendon stiffness observed after SS in this study might have been caused by the decrease in muscle stiffness. In contrast to the data for SS, another recent study revealed that 4 30-s sets of DS in which antagonist muscle groups were contracted did not affect passive muscle-tendon unit stiffness [19], whereas 430 -s sets of DS in which agonist muscles were contracted decreased passive stiffness [9]. Moreover, Samukawa et al. [24] observed proximal displacement of the muscle-tendon junction of the medial gastrocnemius, but no change in the pennation angle or fascicle length after 530 -s sets of DS in which antagonist muscle groups were contracted. Given their findings, the researchers suggested that DS primarily affects the tendinous tissues. Taken together, these previous studies indicate that SS and DS might affect passive muscle-tendon unit stiffness in different ways. Although many studies have examined the effect of SS on passive stiffness, further studies are required to investigate the impact of DS.

We employed the same measurement parameters used in previous studies to investigate PT at the onset of pain according to the pain threshold or stretch tolerance $[7,15]$. Previous authors have proposed that the SS-induced increase in stretch tolerance is caused by a reduction in pain and discomfort perception accompanied by changes in neural and psychological factors, although the detailed mechanisms are unknown [5]. As with SS, DS has been found to significantly increase PT at the onset of pain, and our data were consistent with those of previous studies [19]. Therefore, the increase in PT at the onset of pain after DS might be caused by the same mechanism as that after SS. 
Contrary to our expectations, we did not find any differences in the effects of SS and DS on any of the dependent variables. Moreover, the ES values for the between-post-stretching comparison were negligible or small for measurements with the exception of ROM and PT at the onset of pain. Specifically, the between-poststretching comparison of ROM and PT at the onset of pain showed a medium ES, although these were not statistically significant. These results indicate that the amount of stretching stimulation does not differ between SS and DS. Our stretching protocol might have contributed to these results because the 300 -s stretching duration used in this study was relatively longer than that commonly performed. Therefore, the effects of SS and DS may differ when a shorter stretching duration is employed. Further studies are required to determine whether specific SS and DS protocols have a differential influence on flexibility and muscle performance.

Previous studies have reported that reduced muscle flexibility [30] and increased stiffness [28] are associated with a greater risk of muscle injury. Therefore, we speculate that a total of $300 \mathrm{~s}$ of active static and dynamic stretching may reduce the risk of injury in healthy individuals during sports activities. Indeed, the present results show that both static and dynamic stretching significantly increased ROM and PT at the onset of pain and significantly decreased passive stiffness, indicating that they are both useful preventative measures against injury when preparing to engage in athletic activity.

The main limitation of this study was that we assessed only the effects of a longer duration of stretching (a total of $300 \mathrm{~s}$ for each stretching type). We suspect that it would be difficult to perform $300 \mathrm{~s}$ of stretching per muscle as part of a regular stretching program. Therefore, future studies should compare the effects of static and dynamic stretching with shorter durations of stretching that are more commonly performed (20-60 s). Another limitation was that we collected only isometric muscle force as a measure of muscle performance and not other measures, such as electromyography and muscle contraction speed (rate of force development).

In summary, in the present study, we found that both SS and DS significantly increased ROM and PT at the onset of pain and significantly decreased passive stiffness and isometric muscle force. Interestingly, SS and DS did not differ in terms of the magnitude of change for all measurements. These results suggest that a total of 300 s of SS or DS increases flexibility and decreases isometric muscle force, and that the effects of stretching do not differ between the two stretching methods.

\section{Acknowledgements}

The authors thank Angela Morben, DVM, ELS, Ryan Chastain-Gross, PhD, and Sydney Koke, MFA, from Edanz Group (www.edanzediting.com/ac) for editing a draft of this manuscript. All the authors declare that there are no potential conflicts of interest. This work was supported by JSPS KAKENHI Grant Numbers JP26750288 and JP16K01503.

\section{Conflict of Interest}

The authors declare that they have no conflict of interest.

\section{References}

[1] Amiri-Khorasani M, Sotoodeh V. The acute effects of combined static and dynamic stretch protocols on fitness performances in soccer players. J Sports Med Phys Fitness 2013; 53: 559-565

[2] Behm DG, Blazevich AJ, Kay AD, McHugh M. Acute effects of muscle stretching on physical performance, range of motion, and injury incidence in healthy active individuals: A systematic review. Appl Physiol Nutr Metab 2016; 41: 1-11

[3] Behm DG, Chaouachi A. A review of the acute effects of static and dynamic stretching on performance. Eur J Appl Physiol 2011; 111: 2633-2651

[4] Behm DG, Plewe S, Grage P, Rabbani A, Beigi HT, Byrne JM, Button DC. Relative static stretch-induced impairments and dynamic stretchinduced enhancements are similar in young and middle-aged men. Appl Physiol Nutr Metab 2011; 36: 790-797

[5] Folpp H, Deall S, Harvey LA, Gwinn T. Can apparent increases in muscle extensibility with regular stretch be explained by changes in tolerance to stretch? Aust J Physiother 2006; 52: 45-50

[6] Fowles JR, Sale DG, MacDougall JD. Reduced strength after passive stretch of the human plantarflexors. J Appl Physiol 2000; 89: $1179-1188$

[7] Halbertsma JP, van Bolhuis Al, Goeken LN. Sport stretching: Effect on passive muscle stiffness of short hamstrings. Arch Phys Med Rehabil 1996; 77: 688-692

[8] Harriss D], Macsween A, Atkinson G. Standards for ethics in sport and exercise science research: 2018 update. Int J Sports Med 2017; 38: $1126-1131$

[9] Herda T], Herda ND, Costa PB, Walter-Herda AA, Valdez AM, Cramer JT. The effects of dynamic stretching on the passive properties of the muscle-tendon unit. J Sports Sci 2013; 31: 479-487

[10] Hough PA, Ross EZ, Howatson G. Effects of dynamic and static stretching on vertical jump performance and electromyographic activity. J Strength Cond Res 2009; 23: 507-512

[11] Kataura S, Suzuki S, Matsuo S, Hatano G, Iwata M, Yokoi K, Tsuchida W, Banno Y, Asai Y. Acute effects of the different intensity of static stretching on flexibility and isometric muscle force. J Strength Cond Res 2017; 31: 3403-3410

[12] Kay AD, Blazevich AJ. Moderate-duration static stretch reduces active and passive plantar flexor moment but not Achilles tendon stiffness or active muscle length. J Appl Physiol 2009; 106: 1249-1256

[13] Kay AD, Blazevich AJ. Effect of acute static stretch on maximal muscle performance: A systematic review. Med Sci Sports Exerc 2012; 44: 154-164

[14] Little T, Williams AG. Effects of differential stretching protocols during warm-ups on high-speed motor capacities in professional soccer players. J Strength Cond Res 2006; 20: 203-207

[15] Magnusson SP, Simonsen EB, Aagaard P, Sorensen H, Kjaer M. A mechanism for altered flexibility in human skeletal muscle. J Physiol 1996; 497: 291-298

[16] Matsuo S, Suzuki S, Iwata M, Banno Y, Asai Y, Tsuchida W, Inoue T. Acute effects of different stretching durations on passive torque, mobility, and isometric muscle force. J Strength Cond Res 2013; 27: 3367-3376 
[17] Matsuo S, Suzuki S, Iwata M, Hatano G, Nosaka K. Changes in force and stiffness after static stretching of eccentrically damaged hamstrings. Eur J Appl Physiol 2015; 115: 981-991

[18] Mizuno T, Matsumoto M, Umemura Y. Viscoelasticity of the muscletendon unit is returned more rapidly than range of motion after stretching. Scand J Med Sci Sports 2013; 23: 23-30

[19] Mizuno T, Umemura Y. Dynamic stretching does not change the stiffness of the muscle-tendon unit. Int J Sports Med 2016; 37 : 1044-1050

[20] Nakamura M, Ikezoe T, Takeno Y, Ichihashi N. Acute and prolonged effect of static stretching on the passive stiffness of the human gastrocnemius muscle tendon unit in vivo. J Orthop Res 2011; 29: 1759-1763

[21] Paradisis GP, Pappas PT, Theodorou AS, Zacharogiannis EG, Skordilis EK, Smirniotou AS. Effects of static and dynamic stretching on sprint and jump performance in boys and girls. J Strength Cond Res 2014; 28: 154-160

[22] Perrier ET, Pavol M], Hoffman MA. The acute effects of a warm-up including static or dynamic stretching on countermovement jump height, reaction time, and flexibility. J Strength Cond Res 2011; 25: 1925-1931

[23] Ryan ED, Beck TW, Herda T], Hull HR, Hartman MJ, Stout JR, Cramer JT. Do practical durations of stretching alter muscle strength? A dose-response study. Med Sci Sports Exerc 2008; 40: 1529-1537

[24] Samukawa M, Hattori M, Sugama N, Takeda N. The effects of dynamic stretching on plantar flexor muscle-tendon tissue properties. Man Ther 2011; 16: 618-622
[25] Simic L, Sarabon N, Markovic G. Does pre-exercise static stretching inhibit maximal muscular performance? A meta-analytical review. Scand J Med Sci Sports 2013; 23: 131-148

[26] Smith CA. The warm-up procedure: To stretch or not to stretch. A brief review. J Orthop Sports Phys Ther 1994; 19: 12-17

[27] Trajano GS, Seitz L, Nosaka K, Blazevich AJ. Contribution of central vs. peripheral factors to the force loss induced by passive stretch of the human plantar flexors. J Appl Physiol 2013; 115: 212-218

[28] Watsford ML, Murphy AJ, McLachlan KA, Bryant AL, Cameron ML, Crossley KM, Makdissi M. A prospective study of the relationship between lower body stiffness and hamstring injury in professional Australian rules footballers. Am J Sports Med 2010; 38: 2058-2064

[29] Wilkes-Gillan S, Bundy A, Cordier R, Lincoln M, Chen YW. A randomised controlled trial of a play-based intervention to improve the social play skills of children with attention deficit hyperactivity disorder (ADHD). PloS one 2016; 11: e0160558

[30] Witvrouw E, Danneels L, Asselman P, D'Have T, Cambier D. Muscle flexibility as a risk factor for developing muscle injuries in male professional soccer players. A prospective study. Am J Sports Med 2003; 31: 41-46

[31] Woods K, Bishop P, Jones E. Warm-up and stretching in the prevention of muscular injury. Sports Med 2007; 37: 1089-1099

[32] Yamaguchi T, Ishii K. Effects of static stretching for 30 seconds and dynamic stretching on leg extension power. J Strength Cond Res 2005; 19: 677-683

[33] Yamaguchi T, Ishii K. An optimal protocol for dynamic stretching to improve explosive performance. J Phys Fitness Sports Med 2014; 3: $121-129$ 